\title{
Canadian pandemic influenza preparedness: Public health measures strategy
}

\author{
B Henry ${ }^{1}$ on behalf of the Canadian Pandemic Influenza Preparedness Task Group
}

\begin{abstract}
Public health measures, also known as non-pharmaceutical interventions, are basic actions aimed at slowing the community spread of a communicable disease outbreak. In the event of an influenza pandemic, public health measures and antiviral drugs are the only tools available to mitigate the effects of the pandemic during the months before a vaccine becomes available. The Canadian Pandemic Influenza Preparedness: Planning Guidance for the Health Sector (CPIP) outlines how federal, provincial and territorial governments will work together to ensure a coordinated and consistent health sector approach to pandemic influenza preparedness and response.

This article summarizes Canada's pandemic public health measures strategy, as described in the recently updated CPIP Public Health Measures Annex. The strategy builds on lessons learned during the $2009 \mathrm{H} 1 \mathrm{~N} 1$ pandemic. Key elements of the public health measures strategy include individual measures (e.g. hand hygiene, self-isolation when ill), community-based measures (e.g. school closures, cancellation of mass gatherings), management of cases and close contacts, travel and border-related actions and public education. Factors that influence the effectiveness of public health measures in a pandemic include the pandemic epidemiology, timing of implementation, how the measures are used (i.e. alone or in combination), their scalability and flexibility and public compliance. The CPIP is an evergreen guidance document and the Annex will be updated as new information warrants.
\end{abstract}

This work is licensed under a Creative Commons Attribution 4.0 Internationa License.

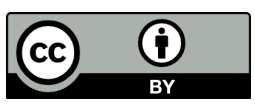

Affiliation

${ }^{1}$ Office of the Provincial Health Officer, Victoria, BC

*Correspondence:

phac.cpip.tg.secretariat-gt.pcp. aspc@canada.ca

Suggested citation: Henry B on behalf of the Canadian Pandemic Influenza Preparedness Task Group. Canadian pandemic influenza preparedness: Public health measures strategy. Can Commun Dis Rep 2019;45(6):159-63.

https://doi.org/10.14745/ccdr.v45i06a03

Keywords: influenza, pandemic, public health measures, non-pharmaceutical interventions

\section{Introduction}

Canada has a multifaceted approach to pandemic influenza preparedness and response that includes the use of public health measures. These measures are the primary non-pharmaceutical means to slow the rate of viral transmission. They are implemented at the beginning of a pandemic before a vaccine becomes available. Public health measures include individual protective measures (e.g. hand hygiene, self-isolation when ill) and community measures (e.g. school closures, cancellation of mass gatherings) to international border and travel-related actions (e.g. entry and departure screening).

The 2009 H1N1 influenza pandemic provided the opportunity for the first real test of Canada's pandemic planning efforts and provided many valuable lessons for future pandemic planning and response. It reinforced that public health measures are an effective and important element of a pandemic response. In addition, there were many valuable lessons to do with planning and implementing public health measures, for example, the importance of implementing measures early in the pandemic in a targeted and layered manner, and providing the public with clear and consistent messaging to support compliance with recommended measures.

The renewed approach to the use of public health measures in an influenza pandemic is described in the Public Health Measures Annex of the Canadian Pandemic Influenza Preparedness: Planning Guidance for the Health Sector $(1,2)$. The Public Health Measures Annex provides technical advice for federal/ provincial/territorial ministries of health and other government departments that have roles in providing health care services to specific populations, such as some Indigenous communities and military personnel. It underscores the importance of all 
levels of government working together to help ensure the public's understanding and adoption of measures. The Annex outlines the specific roles and responsibilities of those involved in selecting and implementing individual and community-level measures aimed at preventing, controlling and mitigating pandemic influenza. The Annex is meant to facilitate a common approach to public health measures across jurisdictions (2).

The Canadian Pandemic Influenza Preparedness (CPIP) Task Group updated the Annex to incorporate the experience gained during the $2009 \mathrm{H} 1 \mathrm{~N} 1$ influenza pandemic and to reflect Canada's pandemic goals, as outlined in the CPIP: "First, to minimize serious illness and overall deaths, and second, to minimize societal disruption" (2). The CPIP is an evergreen guidance document and the Annex will be updated as new information warrants. The Annex should be read in conjunction with the main body and the other technical annexes of the CPIP as they are intended to be used together.

This article summarizes Canada's pandemic public health measures strategy, as described in the recently updated CPIP Public Health Measures Annex (2). It is part of a series outlining Canada's approach to pandemic influenza preparedness published in the Canada Communicable Disease Report (3-8).

\section{Objectives of Canada's pandemic public health measures strategy}

The objectives of the public health measures strategy are to support Canada's pandemic goals by:

- Reducing the overall transmission and slowing the rate of transmission of the novel or pandemic virus, thus lowering the number of severely ill cases and deaths and dispersing the accumulation of cases over time

- Reducing the peak demands on health care institutions to protect against both societal disruption and overwhelming of community services, and to buy time until a vaccine is available

\section{Canadian context}

During an influenza pandemic, collaboration by all levels of government on the approach to selection and use of public health measures is crucial to the success of the response. In Canada, provinces, territories and local public health have the authority to implement public health measures within their jurisdictions. The federal government is responsible for international border and travel-related measures, as well as recommending public health measures for specific populations who are beneficiaries of federal health programs and services, such as some Indigenous communities and correctional facility inmates.
Canada is diverse in terms of language, religious beliefs, ethnicity, culture and lifestyle. In some cases, the needs of the population are not fully addressed by existing services. The CPIP highlights the need for health planners to tailor approaches for individuals who are unable to access and use standard resources and whose circumstances can influence their vulnerability in an influenza pandemic (e.g. persons who have physical or mental disabilities, low literacy skills, a limited understanding of English or French or income insecurity).

It is also important to tailor approaches to the circumstances (e.g. social, environmental, economic, access to health care) of individuals living in remote and isolated communities in pandemic planning. The Annex identifies the unique challenges faced by some of these communities (e.g. limited access to non-medical supplies such as soap, food, household items) and potential strategies for implementing public health measures in these communities (e.g. increasing individual and community awareness and the need to have surge capacity of these basic supplies).

\section{Key elements of the public health measures strategy}

Public health measures are the most basic actions that can be taken to reduce community transmission of a pandemic influenza virus. The measures used in an influenza pandemic are based on existing practices for respiratory infectious disease, modified and intensified based on the epidemiology of the virus. During a pandemic, public health measures are implemented by many people in a variety of settings; therefore, the context in which they are used is important.

The CPIP identifies triggers for action, such as the use of public health measures, based on the level of pandemic virus activity in Canada. It is expected that the selection and timing of public health measures will vary across jurisdictions, as pandemic virus activity will vary across Canada, in terms of the timing and intensity of pandemic waves.

Factors that influence the effectiveness of public health measures in a pandemic response include the pandemic epidemiology (e.g. virus characteristics), timing of implementation, the approach to their use (i.e. alone or in combination) and public compliance. Measures need to be scalable, flexible and proportional to the pandemic threat to optimize their effectiveness.

\section{Individual measures}

During a pandemic, personal protective measures protect individuals, their families and their communities. These measures are at the core of good public health practice for influenza and other respiratory illnesses, and are routinely recommended. Most of these measures are applicable to any pandemic scenario, irrespective of expected impact (ranging from low to high) based on the level of virus transmissibility and clinical severity. 
Individual measures include:

- Hand hygiene (i.e. hand washing or hand antisepsis)

- Respiratory etiquette (i.e. covering sneezes and coughs)

- Cleaning and disinfecting of commonly used surfaces in the living environment

- The use of surgical masks by individuals with influenza

- Voluntary self-isolation (i.e. the sick person isolates themselves from other people from the time that influenza symptoms are recognized and for at least 24 hours after symptoms resolve)

- Voluntary home quarantine (i.e. the exposed person stays at home from the time of their initial exposure for up to three days after their last exposure)

Self-isolation and home quarantine can have unintended secondary consequences, for example, intolerance of employers regarding absences and loss of income if paid leave is not available. The Annex proposes possible strategies for addressing these challenges.

\section{Community-based measures}

Community-based measures are disease control strategies aimed at reducing and slowing the transmission of influenza in communities. Decisions about implementing these measures will likely be made at the level of the local public health authority with coordination at the provincial/territorial or regional levels to ensure a consistent approach. The use of community-based measures will depend on the pandemic impact scenario as well as the local context. Community-based measures include:

- Environmental cleaning of public spaces in keeping with usual pre-pandemic practices (e.g. cleaning products used, surfaces cleaned)

- Social distancing measures that limit the frequency and duration of close contact among individuals of all ages in settings where people congregate, such as workplaces, daycares, schools, shelters, spiritual or cultural settings and mass gathering venues (e.g. concerts, sporting events)

Social distancing measures may have unintended secondary consequences for individuals, families and communities, such as loss of income, an elevated need for support services and potentially reduced availability of certain services. During a lower-impact pandemic scenario, the infection control benefits of implementing some community measures (e.g. proactive school closures) may not be offset by the cost and societal disruption caused by these measures. Hence, such measures are likely to be implemented only during a higher-impact pandemic scenario or in certain situations in some communities.

\section{Travel and border-related measures}

The response to an emerging influenza pandemic includes the use of public health measures targeted to international travellers arriving in or departing from Canada. Such measures are based on existing federal programs and procedures. These include providing education, issuing travel health advisories and administering the Quarantine Act (9) at all international points of entry (e.g. implementing control measures with arriving or departing international travellers or conveyances). At the time of a pandemic, many factors will inform decisions about border measures, including the characteristics of the virus (e.g. transmissibility, virulence, risk factors), evidence of the effectiveness of the measures and the risk to the traveller and the public.

In accordance with the Quarantine Act (9), all ill travellers are subject to entry and departure screening at Canada's borders. Currently, identifying cases of novel or pandemic influenza at points of entry is difficult owing to the similarity of influenza symptoms to various other respiratory viruses. Departure screening is more effective than entry screening at decreasing influenza transmission because it reduces the number of ill travellers boarding conveyances.

\section{Case and contact management measures}

When a novel or pandemic influenza virus is first detected in Canada or elsewhere, public health activities that aim to prevent or limiting the spread of the virus in Canada are triggered. Some circumstances may warrant public health management of cases of novel influenza and their close contacts (e.g. monitoring of health, providing advice on when to seek care).

Case and contact management by public health authorities are likely to be implemented most aggressively in the early stages of a pandemic, before transmission is sustained, in an effort to contain or delay the spread of the virus. If virus transmission is sustained, management activities will shift from individual management to public education in order to reinforce individual and community measures.

\section{Public education}

During an influenza pandemic, public demand for information is expected to be extremely high and sustained as the illness spreads across Canada and into local communities. In the early stages of a pandemic, before a vaccine is available, public health measures are the primary means to slow transmission. Timely public education campaigns, grounded in a risk communications approach, are critical to promoting and supporting the implementation and adoption of measures at the individual and community levels. The strategies, interventions and products developed for seasonal influenza campaigns can be used as the building blocks for pandemic-related education campaigns. Communications will need to be tailored to reach individuals whose needs are not addressed by standard services or who are not able to access resources, which may increase their vulnerability in a pandemic.

\section{Risk management approach}

Canada's pandemic public health measures strategy is subject to numerous risks, including the possibility of unintended secondary consequences of measures and uncertainty of adequate public 
uptake of such measures. The Annex incorporates the CPIP risk management approach to support scalable and flexible pandemic planning by identifying risks and the proposing mitigation. Timely and transparent risk communications to the public and health care providers should be an integral part of the response to each event. Table 1 provides an example of how the CPIP risk management approach is applied to the public health measures strategy.

Table 1: Risks affecting the public health measures strategy, their implications and potential mitigation or response

\begin{tabular}{|l|l|l|}
\hline \multicolumn{1}{|c|}{ Factor/event } & \multicolumn{1}{|c|}{ Implications } \\
$\begin{array}{l}\text { Media report severe illness or a } \\
\text { large number of cases }\end{array}$ & $\begin{array}{l}\text { Sudden increase in demand for information about } \\
\text { public health measure efficacy } \\
\text { Need for implementation of more public health } \\
\text { measures (or perhaps more targeted measures) }\end{array}$ & $\begin{array}{l}\text { Potential mitigation/response } \\
\text { individual public health measures and their rationales } \\
\text { (e.g. hand hygiene, respiratory etiquette, voluntary } \\
\text { self-isolation when ill, environmental cleaning, } \\
\text { caring for the ill, seeking medical assessment). Use } \\
\text { tailored approaches to communicate with vulnerable } \\
\text { populations } \\
\text { Explain to the public how each additional measure } \\
\text { increases personal and group protection and prevention } \\
\text { Advise the public that measures may change as new } \\
\text { information becomes available }\end{array}$ \\
\hline $\begin{array}{l}\text { Differences in implementation of } \\
\text { public health measures between } \\
\text { jurisdictions and internationally }\end{array}$ & $\begin{array}{l}\text { Selection and implementation of public health } \\
\text { measures differ depending on local or regional } \\
\text { situations } \\
\text { Public perception that another jurisdiction's approach } \\
\text { is better } \\
\text { Public concern if there is a perception that public } \\
\text { health resources are inequitably distributed }\end{array}$ & $\begin{array}{l}\text { Acknowledge differences in local or regional } \\
\text { approaches and provide rationale } \\
\text { Ensure that public health measures benefit all groups } \\
\text { within a community or region and that burdens are } \\
\text { equitably distributed }\end{array}$ \\
\hline
\end{tabular}

Source: Pan-Canadian Public Health Network Council. Public Health Measures Annex: Canadian Pandemic Influenza Preparedness: Planning guidance for the health sector (2)

\section{Discussion}

Canada's pandemic influenza preparedness and response requires a multifaceted approach, with public health measures an essential component throughout a pandemic. Public health measures, which require individual and community action to implement, are the most basic actions that can be taken to reduce transmission of a pandemic influenza virus; they are already used across Canada during outbreaks of seasonal influenza and other communicable diseases. Public health measures seek to reduce the occurrence and duration of human infections so as to delay the peak of pandemic influenza activity.

It is important for health planners to take into account that public health measures should be implemented early in a pandemic, in a targeted and layered manner. A communication approach that is transparent and consistent helps to ensure public trust and adoption of recommended measures. The potential benefits of specific measures need to be weighed against the practicality and feasibility of their implementation and potential unintended secondary social and economic impacts. Measures need to tailored for populations and settings whose circumstances may increase their vulnerability in an influenza pandemic.

\section{Conclusion}

The guidance in the CPIP Public Health Measures Annex is intended to promote a scalable and consistent approach to pandemic planning. It is adaptable to different settings. Many of the recommended measures are contingent upon local triggers; therefore, the timing of their implementation depends on local circumstances that may not occur simultaneously across Canadian jurisdictions. However, a consistent approach to applying public health measures and the accompanying messaging will improve public perception, trust and adoption of guidance.

\section{Authors' statement}

Canadian Pandemic Influenza Preparedness Task Group comprises B Henry (Chair), C Alfieri, I Gemmill, T Hatchette, E Henry, S Hota, A Lebans and B Schwartz.

\section{Conflict of interest}

None.

\section{Acknowledgements}

Many thanks to the Public Health Agency of Canada's Immunization Programs and Pandemic Preparedness Division for their contribution to the preparation of this summary: E Henry (Director), L Paddle, S Smith, J Williams, F Lalonde and O Baclic, and to $S$ Rempel, who contributed to the first draft. 


\section{Funding}

The work of the Canadian Pandemic Influenza Preparedness Task Group is supported by the Public Health Agency of Canada.

\section{References}

1. Pan-Canadian Public Health Network Council. Canadian Pandemic Influenza Preparedness: Planning guidance for the health sector. Ottawa: Public Health Agency of Canada; 2018. https://www.canada.ca/en/public-health/services/ flu-influenza/canadian-pandemic-influenza-preparednessplanning-guidance-health-sector.html

2. Pan-Canadian Public Health Network Council. Public Health Measures Annex: Canadian Pandemic Influenza Preparedness: Planning guidance for the health sector. Ottawa: Public Health Agency of Canada; 2019. https://www. canada.ca/en/public-health/services/flu-influenza/canadianpandemic-influenza-preparedness-planning-guidance-healthsector/public-health-measures.htm

3. Henry B, Gadient S; Canadian Pandemic Influenza Preparedness (CPIP) Task Group. Canada's pandemic vaccine strategy. Can Commun Dis Rep 2017 Jul;43(7/8):164-7.

DOI PubMed
4. Henry B; Canadian Pandemic Influenza Preparedness (CPIP) Task Group. Canada's Pandemic Influenza Preparedness: laboratory strategy. Can Commun Dis Rep 2018 Jan;44(1):10-3. DOI PubMed

5. Henry B; Canadian Pandemic Influenza Preparedness (CPIP) Task Group. Canada's pandemic influenza preparedness: surveillance strategy. Can Commun Dis Rep 2018 Jan;44(1):14-7. DOl PubMed

6. Henry B; Canadian Pandemic Influenza Preparedness (CPIP) Task Group. Canadian Pandemic Influenza Preparedness: health sector planning guidance. Can Commun Dis Rep 2018 Jan;44(1):6-9. DOI PubMed

7. Henry B; Canadian Pandemic Influenza Preparedness (CPIP) Task Group. Canadian pandemic influenza preparedness: communications strategy. Can Commun Dis Rep 2018 May;44(5):106-9. DOI PubMed

8. Henry B; Canadian Pandemic Influenza Preparedness (CPIP) Task Group. Canadian pandemic influenza preparedness: antiviral strategy. Can Commun Dis Rep 2019 Jan;45(1):3843. DOI PubMed

9. Department of Justice. Quarantine Act, RSC 2005, c20, s82. Repeals RSC 1985, cQ-1. Ottawa: Government of Canada. (Accessed 2019-03-05). https://laws-lois.justice.gc.ca/eng/ acts/q-1.1/page-1.html 